\title{
Channel Selection under Interference Temperature Model in Multi-hop Cognitive Mesh Networks
}

\author{
Manuj Sharma ${ }^{*}$, Anirudha Sahoo*, and K. D. Nayak ${ }^{\#}$ \\ *Kanwal Rekhi School of Information Technology, \\ IIT Bombay, Powai, Mumbai, India \\ email: \{manuj, sahoo\}@it.iitb.ac.in
\#Advanced Numerical Research and Analysis Group, Defence Research \& Development Organization, Hyderabad, India

\begin{abstract}
A cooperative algorithm based on interference temperature model is proposed for computation of available channels by mesh nodes in a cognitive mesh network. Interference temperature model is used to model occupancy and availability of a channel. Link and end-to-end routing metrics are proposed to select appropriate channels from the computed set of available channels.
\end{abstract}

\section{INTRODUCTION}

$\mathrm{S}$ haring of licensed spectrum is a promising approach to utilize spectrum resource in wireless communication. One of the proposed mechanisms to enable such sharing is to use Interference Temperature Model. Under this model, unlicensed devices are allowed to use channels in licensed spectrum bands in a geographical region for their transmissions, provided the interference temperature (see section II for definition of interference temperature) at each licensed receiver in the region does not exceed a predefined interference temperature threshold [1]. The regulatory bodies need to define these thresholds for all the channel frequencies in various geographical regions.

In this paper, we describe algorithms for selecting the most appropriate channel for transmission under interference temperature model by a multi-interface node in a cognitive mesh network. We consider both the fixed and adaptive transmission power cases. Each mesh node computes a set of channels available for transmission (without increasing the interference temperature beyond the threshold in its interference range). It then uses a per-hop link cost metric and end-to-end routing metric (described in section IV) to select channels from the available channels set for each hop on the end-to-end path. We define and introduce a novel channel stability factor in per-hop link cost calculation for each channel.

The rest of the paper is organized as follows. Section II describes some preliminary concepts used in this paper. Section III presents the notations and algorithms for computing the available channel set for both the fixed and adaptive transmission power cases. In section IV, we propose link and end-to-end routing metrics to select channels for endto-end path hops. Section V briefly highlights the possible future work.

\section{PRELIMINARIES}

\section{A. Interference Temperature}

Interference temperature is defined as a measure of the $\mathrm{RF}$ power available at a receiving antenna to be delivered to a receiver. This is the power generated by other emitters and noise sources. More specifically, it is the temperature equivalent of the RF power available at a receiving antenna per unit of bandwidth, measured in units of Kelvin [2, 3]. Interference temperature is calculated as [4]:

$$
T_{c}\left(f_{c}, B_{c}\right)=\frac{P_{i}\left(f_{c}, B_{c}\right)}{k B_{c}}
$$

where $T_{c}\left(f_{c}, B_{c}\right)$ is the interference temperature for channel $c$, with central frequency $f_{c}$ and bandwidth $B_{c} . P_{i}\left(f_{c}, B_{c}\right)$ is the average interference power in Watts (at the antenna of a receiving or measuring device) centered at frequency $f_{c}$ and covering the bandwidth $B_{c}$ (in Hertz). $k$ is Boltzmann's constant $\left(1.38 \times 10^{-23}\right.$ Joules per Kelvin $)$.

\section{B. Cognitive Mesh Network}

A cognitive radio is a smart radio which has the ability to sense the external environment, learn from the history, and make intelligent decisions to adjust its transmission parameters according to the current state of environment. A cognitive mesh network is a static, multi-hop wireless mesh network in which each node is equipped with multiple cognitive radios. In this paper, we consider a multi-channel mesh in which each mesh node measures the interference temperature for each channel locally, and exchanges these measured values with the other nodes within its interference range. Based on the collected statistics, it derives the interference temperature for each channel at various locations in that area.

Under interference temperature, we define a channel $c$ (with central frequency $f_{c}$ and bandwidth $\mathrm{B}_{\mathrm{c}}$ ) to be available for transmission at a mesh node $m$ if the transmission due to $m$ does not increase the interference temperature at any other mesh node $n$ (within the interference range of $m$ ) beyond a predefined threshold. The above constraint is formally represented as [4]:

$$
T_{c}\left(f_{c}, B_{c}\right)+\frac{L_{m n}^{c} P t_{m}\left(f_{c}, B_{c}\right)}{k B_{c}}<T H_{c}
$$


Here, $L_{m n}^{c}$ is the path loss in transmission from node $m$ to $n$ on channel $c, P t_{m}\left(f_{c}, B_{c}\right)$ is the transmission power of $m$, and $T H_{c}$ is the interference temperature threshold for channel $c$. Other symbols are same as defined in (1). Channel availability can be modeled using a simple 2-state Markov chain (shown in Figure 1). States $A$ and $B$ represents a channel being available and unavailable respectively at a mesh node. Symbols $\beta_{c}$ and $\mu_{c}$ represents respectively the probability with which the interference temperature for a channel $c$ overshoots (or drops-down under) the predefined threshold (thereby making the channel $c$ transit from the state of availability to that of unavailability, and vice versa, respectively). The sojourn time of each state is geometrically distributed. Therefore, the sojourn time of state $A$, which denotes the average time for which channel $c$ remains available, is given as:

$$
\tau_{c}^{A}=1 /\left(1-\beta_{c}\right)
$$

We propose to use $\tau_{c}^{A}$ as one of the parameters in routing metric to select the most appropriate channel from the set of all the available channels. The challenge in the above model lies in calculating the transition rates $\left(\beta_{c}\right.$ and $\left.\mu_{c}\right)$ of interference temperature value (for channel $c$ ) across the threshold.

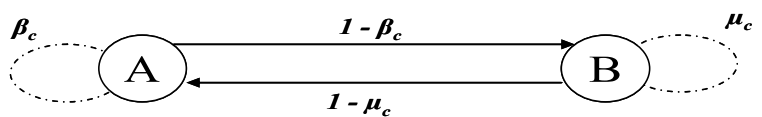

Fig. 1. A 2-state Markov chain to model a channel.

\section{AVAILABLE CHANNEL SET COMPUTATION ALGORITHM}

\section{A. Notations}

$N$ : Set of all the mesh nodes, $\{1 . . \mathrm{N}\}$.

$C$ : Set of all the channels (frequency bands) in the spectrum pool, $\{1 . . \mathrm{C}\}$.

$\mathrm{m}$ : A mesh node, which is under consideration for computing the available channel set for sending its data.

$T R_{m}$ :Set of mesh nodes which are within the transmission range of node $\mathrm{m}$.

r: Receiver mesh node (within the transmission range of node $\mathrm{m}$ ) that has to receive data from sender node $\mathrm{m}$. $\left(\mathrm{r} \in T R_{m}\right)$.

$I R_{m}$ : Set of mesh nodes which are within the interference range of node $\mathrm{m}$. Note that, $\mathrm{j} \in T R_{m} \Rightarrow \mathrm{j} \in I R_{m}$.

$\mathrm{n}$ : A mesh node within the interference range of node $\mathrm{m}$, i.e. $\mathrm{n} \in I R_{m}$.

SIR_Th: The threshold for signal-to-interference ratio at a node $\mathrm{j}$ (where $\mathrm{j} \in N$ ) for which node $\mathrm{j}$ can successfully receive a packet $\mathrm{j}$. It is assumed that $\mathrm{SIR}_{-} \mathrm{Th}_{\mathrm{j}}$ is constant for all the channels $\mathrm{c} \in C$.

$L T_{m}$ : Local interference temperature vector measured by node $\mathrm{m}, \forall \mathrm{c} \in C$.
$L T_{r}$ : Local interference temperature vector measured by node $\mathrm{r}, \forall \mathrm{c} \in C$.

$L T_{n}:$ Local interference temperature vector measured by node $\mathrm{n} \in I R_{m}, \forall \mathrm{c} \in C$.

$\mathrm{LT}_{\mathrm{m}}[\mathrm{c}], \mathrm{LT}_{\mathrm{r}}[\mathrm{c}]$, and $\mathrm{LT}_{\mathrm{n}}[\mathrm{c}]:$ Local interference temperature measured by node $\mathrm{m}, \mathrm{r}$, and $\mathrm{n}$, respectively for channel $\mathrm{c} \in C$.

$\mathrm{V}_{\mathrm{j}}$ : Tuple containing a node's identifier (j), the local interference temperature vectored measured by node $\mathrm{j}$, and SIR_Th ${ }_{j}$; that is, $\left(\mathrm{n}, L T_{j}\right.$, SIR_Th ${ }_{\mathrm{j}}$ ).

$T_{m}^{n}:$ Vector of aggregate interference temperatures for all channels experienced at node $\mathrm{n}$ due to transmissions by node $\mathrm{m}$; it is calculated by node $\mathrm{m}$ by adding its own potential contribution to the existing interference temperature at node $\mathrm{n}$ (for all channels).

$\mathrm{T}_{m}^{n}$ [c]: Aggregate interference temperature for channel c experienced at node $\mathrm{n}$ due to transmissions by node $\mathrm{m}$.

$\mathrm{T}_{m}^{m}[\mathrm{c}]$ : Aggregate interference temperature for channel $\mathrm{c}$ experienced in the neighborhood of $\mathrm{m}$ due to its transmissions; it is calculated by node $\mathrm{m}$ by adding its own potential contribution to the existing interference temperature that it has measured for channel $\mathrm{c}$.

$\mathrm{TH}_{\mathrm{c}}$ : Predefined interference temperature threshold for channel c.

$\alpha$ : Scale-down factor for interference temperature threshold.

$\mathrm{L}_{m n}^{c}$ : Path loss in transmission from node $\mathrm{m}$ to $\mathrm{n}$ on channel c.

$\mathrm{f}_{\mathrm{c}}$ : Central frequency of channel $\mathrm{c}$.

$\mathrm{B}_{\mathrm{c}}$ : $\quad$ Bandwidth of channel $\mathrm{c}$.

$\mathrm{Pt}_{\mathrm{j}}\left(\mathrm{f}_{\mathrm{c}}, \mathrm{B}_{\mathrm{c}}\right)$ : Transmission power of a node $\mathrm{j}$ over channel $\mathrm{c}$, (where $\mathrm{j} \in N$ ).

$\operatorname{Pr}_{\mathrm{j}}\left(\mathrm{f}_{\mathrm{c}}, \mathrm{B}_{\mathrm{c}}\right)$ : Received signal power at a node $\mathrm{j}$ over channel $\mathrm{c}$, (where $\mathrm{j} \in N$ ).

$\mathrm{Pi}_{\mathrm{j}}\left(\mathrm{f}_{\mathrm{c}}, \mathrm{B}_{\mathrm{c}}\right)$ : Aggregate interference power at a node $\mathrm{j}$ over channel $\mathrm{c}$, (where $\mathrm{j} \in N$ ).

$\mathrm{k}$ : Boltzmann constant $\left(1.38 \times 10^{-23}\right.$ Joules per Kelvin $)$.

$P C_{m}$ : Vector of probable channels

$A C_{m}$ : Set of channels available at node $\mathrm{m}$ for transmission without increasing interference temperature beyond defined threshold at any node $\mathrm{n} \in I R_{m}$.

\section{B. Initialization at each mesh node}

1. Compute location coordinates $\left(\mathrm{x}_{\mathrm{i}}, \mathrm{y}_{\mathrm{i}}, \mathrm{z}_{\mathrm{i}}\right) \forall \mathrm{i} \in N$ using any location determination technique.

2. For each node $\mathrm{i} \in N$, exchange $\left(\mathrm{x}_{\mathrm{i}}, \mathrm{y}_{\mathrm{i}}, \mathrm{z}_{\mathrm{i}}\right)$ with all the other nodes $\mathrm{j} \in\left(N \cap I R_{i}\right)$.

3. For each node $i \in N$, compute its distance from every other node in its interference range:

$$
\begin{aligned}
\mathrm{d}_{\mathrm{ij}}=\left(\left(\mathrm{x}_{\mathrm{i}}-\mathrm{x}_{\mathrm{j}}\right)^{2}+\left(\mathrm{y}_{\mathrm{i}}-\mathrm{y}_{\mathrm{j}}\right)^{2}+\right. & \left.\left(\mathrm{z}_{\mathrm{i}}-\mathrm{z}_{\mathrm{j}}\right)^{2}\right)^{1 / 2}, \\
& \forall \mathrm{j} \in\left(N \cap I R_{i}\right) .
\end{aligned}
$$

4. For each node $\mathrm{i} \in N$, calculate $\mathrm{L}_{\mathrm{ij}}^{\mathrm{c}}$ using $\mathrm{d}_{\mathrm{ij}}$, 
$\forall \mathrm{j} \in\left(N \cap I R_{i}\right)$ and $\forall \mathrm{c} \in C$.

After initialization, node $\mathrm{m}$ under consideration will have

$\mathrm{L}_{m n}^{c}$ for all $\mathrm{n} \in I R_{m}$ and all $\mathrm{c} \in C$.

\section{Computation of Available Channel Set: The Fixed Transmission Power Case}

Following are the main steps of the algorithm, assuming that each mesh node transmits with a fixed transmission power.

1) At each mesh node $n \in I R_{m}$ :

1.1 Compute $L T_{n}$.

1.2 Transmit tuple $\mathrm{V}_{\mathrm{n}}=\left(\mathrm{n}, L T_{n}\right)$ to all nodes within its interference range.

2) At mesh node $m$

2.1 Receive $\mathrm{V}_{\mathrm{n}}$ from each node $\mathrm{n} \in I R_{m}$.

2.2 For each $\mathrm{c} \in C$ :

$$
\begin{gathered}
2.2 .1 \quad \mathrm{~T}_{m}^{m}[\mathrm{c}]=\left\{\mathrm{Pt}_{\mathrm{m}}\left(\mathrm{f}_{\mathrm{c}}, \mathrm{B}_{\mathrm{c}}\right) /\left(\mathrm{k} \mathrm{B}_{\mathrm{c}}\right)\right\}+\mathrm{LT}_{\mathrm{m}}[\mathrm{c}] \\
\text { 2.2.2 } \\
\text { If }\left(\mathrm{T}_{m}^{m}[\mathrm{c}] \leq \alpha . \mathrm{TH}_{\mathrm{c}}\right) \text { then } \\
P C_{m}=P C_{m} \cup\{\mathrm{c}\}
\end{gathered}
$$

2.3 For each mesh node $\mathrm{n} \in I R_{m}$ :

$$
\text { 2.3.1. For each } \mathrm{c} \in P C_{m} \text { : }
$$

$$
\begin{gathered}
\text { 2.3.1.1 } \mathrm{T}_{m}^{n}[\mathrm{c}]=\left\{\left(\mathrm{L}_{m n}^{c} * \mathrm{Pt}_{\mathrm{m}}\left(\mathrm{f}_{\mathrm{c}}, \mathrm{B}_{\mathrm{c}}\right)\right) /(\mathrm{k}\right. \\
\left.\left.\mathrm{B}_{\mathrm{c}}\right)\right\}+\mathrm{LT}_{\mathrm{n}}[\mathrm{c}] \\
\text { 2.3.1.2 } \\
\text { If }\left(\mathrm{T}_{m}^{n}[\mathrm{c}] \leq \alpha . \mathrm{TH}_{\mathrm{c}}\right) \text { then } \\
A C_{m}=A C_{m} \cup\{\mathrm{c}\}
\end{gathered}
$$

Every node $n$ within the interference range of node $m$ measures locally the interference temperature for all the channels and periodically sends the measured information to all the nodes within its interference range (statements 1.1 and 1.2 in above algorithm). This dissemination of information can be done on a dedicated 802.11 channel of unlicensed band. For the nodes that are outside the communication range but within the interference range of the sending node, multihop relay communication, or increased transmission power need to be used. Assuming bi-symmetrical channel, node $m$ receives the interference temperature vector for all channels from all other nodes within its interference range (statement 2.1). It then first measures the interference temperature locally for all the channels and calculates the aggregate interference temperature (for all channels) that would be experienced in its neighborhood due to its transmissions (statement 2.2.1). Next, it discards those channels for which interference temperature exceeds the scaled-down threshold value, and keeps the remaining channels in the set of probable channels, $P C_{m}$ (statement 2.2.2.). These channels are probable, because even though they do not increase the interference temperature beyond the threshold in the neighborhood of node $m$, they may increase the temperature beyond the threshold at other mesh nodes which are within the interference range of node $m$. In statement 2.3.1.1, node $m$ computes the aggregate interference temperature experienced by all nodes $n$ within its interference range due its transmission on all the probable channels. Out of all the probable channels, it then selects those channels as available channels $\left(A C_{m}\right)$ for which interference temperature would not exceed the threshold value at any mesh node within its interference range when it (node $\mathrm{m}$ ) transmits on those channels (statement 2.3.1.2). Therefore, available channel set at a node $m$ is essentially the set of those channels on which transmission by $m$ will not increase the aggregate interference temperature beyond a predefined threshold.

\section{Computation of Available Channel Set: The Adaptive Transmission Power Case}

Following are the main steps of the algorithm, assuming adaptive transmission power control for each mesh node.

1) At each mesh node $n \in I R_{m}$ :

1.1 Compute $L T_{n}$.

1.2 Transmit tuple $\mathrm{V}_{\mathrm{n}}=\left(\mathrm{n}, L T_{n}, \mathrm{SIR} \_T \mathrm{~T}_{\mathrm{n}}\right)$ to all nodes within its interference range.

2) At mesh node $m$

2.1 Receive $\mathrm{V}_{\mathrm{n}}$ from each node $\mathrm{n} \in I R_{m}$, including from the intended receiver node $r$.

2.2 For each $\mathrm{c} \in C$ :

2.2.1 Calculate the interference power level experienced by receiver node $\mathrm{r}$ :

$$
\mathrm{Pi}_{\mathrm{r}}[\mathrm{c}]=\mathrm{k} \mathrm{B}_{\mathrm{c}} \mathrm{LT}_{\mathrm{r}}[\mathrm{c}]
$$

2.2.2 Calculate the minimum signal power required (under given interference temperature) at receiver $r$ to receive the packet correctly:

$$
\operatorname{Pr}_{\mathrm{r}}[\mathrm{c}]=\mathrm{k} \mathrm{B}_{\mathrm{c}} \mathrm{LT}_{\mathrm{r}}[\mathrm{c}] \mathrm{SIR} \_\mathrm{Th}_{\mathrm{r}}
$$

2.2.3 Node $\mathrm{m}$ must therefore transmit with the following power:

$$
\mathrm{Pt}_{\mathrm{m}}[\mathrm{c}]=\operatorname{Pr}_{\mathrm{r}}[\mathrm{c}] / \mathrm{L}_{m r}^{c}
$$

2.2.4 $\mathrm{T}_{m}^{m}[\mathrm{c}]=\left\{\mathrm{Pt}_{\mathrm{m}}[\mathrm{c}] /\left(\mathrm{k} \mathrm{B}_{\mathrm{c}}\right)\right\}+\mathrm{LT}_{\mathrm{m}}[\mathrm{c}]$

2.2.5 If $\left(\mathrm{T}_{m}^{m}[\mathrm{c}] \leq \alpha\right.$. $\left.\mathrm{TH}_{\mathrm{c}}\right)$ then

$$
P C_{m}=P C_{m} \cup\{\mathrm{c}\}
$$

2.3 For each mesh node $\mathrm{n} \in I R_{m}$ :

2.3.1. For each $\mathrm{c} \in P C_{m}$ :

$$
\text { 2.3.1.1 } \mathrm{T}_{m}^{n}[\mathrm{c}]=\left\{\left(\mathrm{L}_{m n}^{c} * \mathrm{Pt}_{\mathrm{m}}[\mathrm{c}]\right) /(\mathrm{k}\right.
$$$$
\left.\left.\mathrm{B}_{\mathrm{c}}\right)\right\}+\mathrm{LT}_{\mathrm{n}}[\mathrm{c}]
$$

2.3.1.2 If $\left(\mathrm{T}_{m}^{n}[\mathrm{c}] \leq \alpha\right.$. $\left.\mathrm{TH}_{\mathrm{c}}\right)$ then

$$
A C_{m}=A C_{m} \cup\{\mathrm{c}\}
$$

Here, each transmitter node $m$ uses the value of interference experienced at receiver node $r$, the Signal-to-Interference threshold, and the path loss value (between itself and node r), to calculate the transmission power it must use to transmit data to node $r$ (steps 2.2.1, 2.2.2 and 2.2.3). Once the required transmission power has been calculated by node $m$ (step 2.2.3), remaining steps are similar to those defined for the 
fixed transmission power case (Section III-C).

\section{LINK AND ROUTING METRICS}

\section{A. Per-hop Link Metric for Channel Selection}

Once a node computes the set of all the available channels, it must then select one appropriate channel for transmission to its next hop neighbor. First prerequisite of channel selection for a link between two neighboring nodes is that both the nodes must have a non-empty intersection of their available channel sets, i.e. for neighboring nodes $m$ and $n$ to communicate, $A C_{m} \cap A C_{n} \neq \phi$. Assuming that node $m$ needs to transmit data to node $n$, and the above constraint holds for both the nodes, node $m$ must then select a channel out of the common channel set for the link between itself and node $n$. Two established criteria to select a channel for a link are: (i) the selected channel should take minimum possible amount of time to transmit a link-layer frame from node $m$ to $n$; (ii) it should have minimum possible switching cost. In addition to these parameters, we propose two additional parameters while considering a channel to select: the channel stability factor, $S F_{c}$, and the transmission power $\left(P t_{m}[c]\right)$ required for successful transmission over the channel (only for adaptive power controlled nodes). We define the channel stability factor for a channel $c$ as the average amount of time for which the channel remain available for transmission (i.e. the average amount of time for which its interference temperature remained below the scaled-down threshold $\left.\left(\alpha \cdot \mathrm{TH}_{\mathrm{c}}\right)\right)$. The average channel availability time, $\tau_{c}^{\prime}$, can be calculated as weighted moving average of the previously measured availability times, and the time measured in the current measurement duration $\left(\tau_{c}\right)$, as follows:

$$
\tau_{c}^{\prime}=\gamma \tau_{c}^{\prime}+(1-\gamma) \tau_{c}
$$

where $\gamma$ is the smoothing factor for $\tau_{c}^{\prime}$. As per above definition, we take

$$
\mathrm{SF}_{\mathrm{c}}=\tau_{c}^{\prime}
$$

To account for the transmission time and channel switching parameters mentioned above, we use the Expected Transmission Time (ETT) of a frame and Channel Switching cost (SC) metrics for a channel as defined in [5]. With all the parameters defined, we formulate the cost metric for channel selection for a given link $\left(\mathrm{LC}_{\mathrm{c}}\right)$ as follows.

For fixed transmission power case:

$$
\mathrm{LC}_{\mathrm{c}}=\mathrm{w}_{1} \mathrm{ETT}_{\mathrm{c}}+\mathrm{w}_{2} \mathrm{SC}_{\mathrm{c}}+\mathrm{w}_{3}\left(1 / \mathrm{SF}_{\mathrm{c}}\right)
$$

For adaptive transmission power case:

$$
\mathrm{LC}_{\mathrm{c}}=\mathrm{w}_{1} \mathrm{ETT}_{\mathrm{c}}+\mathrm{w}_{2} \mathrm{SC}_{\mathrm{c}}+\mathrm{w}_{3}\left(1 / \mathrm{SF}_{\mathrm{c}}\right)+\mathrm{w}_{4} \mathrm{Pt}_{\mathrm{m}}[\mathrm{c}]
$$

where $\mathrm{LC}_{\mathrm{c}}$ is cost of a link when channel $\mathrm{c}$ is used, and other symbols are as defined above for channel c. The values $\mathrm{w} 1$, $\mathrm{w} 2, \mathrm{w} 3$, and $\mathrm{w} 4$ are the weights that can be assigned to different parameters under the constraint that $w 1+w 2+w 3+$ $\mathrm{w} 4=1$. The appropriate value for these weights and the basis for deciding them is a subject of further study.

\section{B. End-to-end Routing Metric}

Assuming that the end-to-end route $r$ consists of $p$ hops, we update the routing metric suggested in [5] to use the link cost definition given in eq. (4) or (5). The routing metric is formulized as:

$$
R M(r)=(1-\delta) * \sum_{i=1}^{p} L C c_{i}+\delta *\left(\max _{1 \leq j \leq C} X_{j}\right)
$$

Here, $\mathrm{RM}(\mathrm{r})$ is the routing metric for route $\mathrm{r}, L C c_{i}$ is the cost of using channel $\mathrm{c}$ on hop $\mathrm{i}, X_{j}$ is the total number of times channel $\mathrm{j}$ is used in route $\mathrm{r}$, and $\mathrm{C}$ is the total number of channels in the spectrum pool. $L C c_{i}$ is given by equations (4) or (5) depending upon whether fixed power or adaptive power controlled nodes are assumed in the network.

\section{FUTURE WORK}

Some of the important and interesting areas of future work include: (i) studying the effect of heterogeneous channel set in conjunction with dynamic channel set (which is already considered in this paper), particularly focusing on the protocol stack layer where this channel heterogeneity should be handled [6], (ii) design of higher layer protocols, such as transport layer, for dynamic and heterogeneous channels set, and to formulate appropriate solution for them, and finally, (iii) the design of a virtual MAC layer abstraction that can work with different heterogeneous channels.

\section{REFERENCES}

[1] FCC Notice of Inquiry and Notice of Proposed Rule Making, "In the matter of establishment of an interference temperature metric to quantify and manage interference and to expand available unlicensed operation in certain fixed, mobile and satellite frequency bands," ET Docket No. 03237, November 13, 2003.

[2] FCC Spectrum Policy Task Force, "Report of the Interference Protection Working Group," November 15, 2002.

[3] Paul J. Kolodzy, "Interference temperature: a metric for dynamic spectrum utilization," Wiley Int. J. Network Management, vol. 16, pp. 103-113, 2006.

[4] T. Clancy, "Formalizing the Interference Temperature Model," to appear in Wiley Wireless Communications and Mobile Computing 2006. (Available online at: http://www.cs.umd.edu/ clancy/docs/itmawcmc06.pdf).

[5] Pradeep Kyasanur, and Nitin H. Vaidya, "Routing and link-layer protocols for multi-channel multi-interface ad hoc wireless networks," in ACM Mobile Computing and Communications Review, vol. 1, no. 2, January 2006.

[6] Pradeep Kyasanur, and Nitin H. Vaidya, "Protocol Design Challenges for Multi-hop Dynamic Spectrum Access Networks", Proc. of IEEE DySPAN, November 2005. 